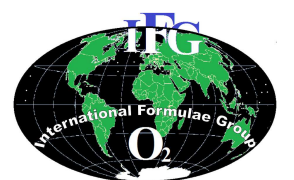

\title{
Influence de la farine des feuilles de Leucaena leucocephala sur les performances de croissance des lapereaux
}

\author{
H.F. DEFANG ${ }^{1 *}$, T.C. KEAMBOU ${ }^{2}$, Y. MANJELI ${ }^{1}$, A. TEGUIA ${ }^{1}$ et T.E. PAMO ${ }^{1}$ \\ ${ }^{I}$ Département des Productions Animales, Faculté d'Agronomie et des Sciences Agricoles, \\ Université de Dschang, Cameroun. \\ ${ }^{2}$ Department of Live Sciences, University of Buea, Cameroon. \\ *Auteur correspondant; E-mail: fdefang@yahoo.com ; B.P:188 Dschang, Cameroun. \\ Tel: (+237)33068129, (+237)99252275
}

\section{RÉSUMÉ}

Quarante (40) lapereaux locale âgés de 8 semaines environ et pesant en moyenne $1000 \pm 200 \mathrm{~g}$ ont été utilisés pour évaluer l'effet de la substitution de 0, 5, 10, 15 et 20\% de tourteau de coton par la farine des feuilles de Leucaena leucocephala dans la ration, suivant un dispositif complètement randomisé. Les animaux étaient logés individuellement et nourris ad libitum. Il ressort des résultats de cet essai que la consommation alimentaire a augmenté significativement $(\mathrm{P}<0,05)$ avec le niveau d'incorporation de la farine de $L$. leucocephala. Cette consommation a été de 90,98; 86,08; 93,00; 107,27 et 116,64 g (MS) respectivement pour les rations à $0,5,10,15$ et $20 \%$ de farine des feuilles de Leucaena leucocephala. Le niveau d'incorporation des feuilles de Leucaena leucocephala n'a eu aucun effet significatif ni sur l'évolution du poids vif, ni sur le gain moyen quotidien et les indices de consommation pendant toute la durée de l'essai. Cependant, la ration contenant $20 \%$ de farine des feuilles de L. leucocephala, s'est avérée plus économique. D'une manière générale, les résultats obtenus laissent penser que le taux d'incorporation de $20 \%$ de $L$ leucocephala donne de meilleures performances et pourrait être recommandé. Toutefois, d'autres investigations sont nécessaires pour déterminer le niveau optimal d'incorporation de L. leucocephala, en évitant les effets néfastes de la mimosine que contiendraient ces feuilles.

() 2014 International Formulae Group. All rights reserved.

Mots clés : Croissance, Leucaena leucocephala, lapereaux.

\section{INTRODUCTION}

Le flux continu d'aide et des importations alimentaires dans les pays en développement en général, et au Cameroun en particulier, est une preuve suffisante que l'autosuffisance alimentaire reste un pari à gagner. Des besoins nutritionnels, ceux des protéines, sont les plus prononcés, et leur carence serait en partie responsable de la recrudescence des maladies nutritionnelles traduites par un taux élevé de mortalité infantile et une relative baisse de l'espérance de vie à la naissance.

S'il est vrai que l'élevage du gros bétail contribue pour environ $80 \%$ des approvisionnements en protéines animales pour toute la population, les petites espèces comme la volaille, les cochons d'inde, les lapins entre autres, qui ont des exigences moindres, sont à la portée de la majorité des 
ménages démunis et pourraient à n'en point douter solutionner le problème de carence en protéine animale chez ces derniers (Bergaoui, 1992). Le lapin peut constituer un revenu non négligeable pour le foyer (Djellal et al., 2006).

Par ailleurs, l'alimentation représentant plus de $70 \%$ des coûts de production, la maîtrise des ressources alimentaires localement disponibles devient un préalable au succès de l'élevage dans les pays en développement. Même chez les espèces pouvant dépendre en majorité des fourrages marginaux, il a été démontré que les supplémentations protéiques pourraient doubler ou tripler leur productivité. Malheureusement, les sous-produits agroindustriels utilisés de façon classique deviennent de plus en plus rares, d'ou la nécessité de recourir aux espèces de légumineuses ne faisant pas l'objet de compétition avec l'Homme, comme le $L$. leucocephala qui se distingue par sa haute valeur nutritive (Jabbar et al., 1997 ; Aregheore et Perera, 2004).

L'objectif de ce travail est de contribuer à l'amélioration de la productivité des lapins locale en utilisant les ressources alimentaires produites localement. Il s'agit plus spécifiquement de déterminer les paramètres zootechniques, d'estimer le taux optimal d'incorporation de la farine des feuilles de L. leucocephala en substitution des tourteaux de coton dans la ration des lapereaux en croissance, et d'évaluer la rentabilité économique de cette opération en vue de retenir la ration qui pourrait être adoptée par des paysans.

\section{MATERIEL ET METHODES}

L'essai a porté sur 40 lapereaux locaux du Cameroun et des deux sexes (20 mâles et 20 femelles) de 8 semaines d'âge environ. Les animaux ont été logés dans des cages métalliques individuelles à plan incliné avec mangeoire et abreuvoir incorporés puis, soumis à 12 jours d'adaptation avant d'être repartis en 5 traitements (rations) de huit sujets (répétitions) dont 4 mâles et 4 femelles par traitement et de poids sensiblement homogènes $(1000 \pm 200 \mathrm{~g}$ en début d'essai). Les feuilles de Leucaena utilisées ont été récoltées par élagage des jeunes ramifications de 1,5 à 2 mois d'âge. Elles ont été par la suite séchées au soleil et stockées jusqu'en début de l'essai. Leur détoxification a été faite par addition de sulfate de fer à $0,6 \%$ directement aux rations expérimentales. Les cinq rations expérimentales (Tableau 1) ont été formulées par incorporation des taux croissants de la farine de L. leucocephala, en substitution au tourteau de coton dans la ration témoin. A chaque traitement, la ration expérimentale correspondante était quantifiée et servie ad libitum, en plus du fourrage. Tous les animaux ont bénéficié du même plan de prophylaxie. Les données de consommation alimentaire, gain de poids, évolution pondérale et indice de consommation ont été obtenues à une fréquence hebdomadaire. L'analyse économique a été faite sur la base du prix des différents ingrédients sur le marché local. Ces données ont été soumises au model linéaire général de l'analyse de la variance. Le test de Duncan a été utilisé pour séparer les moyennes lorsqu'elles présentaient des différences significatives.

\section{RESULTATS}

\section{Consommation alimentaire}

L'analyse de la variance a montré que la consommation alimentaire des lapereaux est significativement influencée $(\mathrm{P}<0,05)$ par le niveau d'incorporation de la farine des feuilles de $L$ leucocephala, dans les rations, notamment à la $4^{\text {ième }}$ semaine de l'essai. La Figure 1 illustre l'évolution de cette consommation. Il ressort de l'analyse de cette figure que la consommation croit à la fois avec l'âge et le niveau d'incorporation de la farine des feuilles de L leucocephala. Ceci serait dû non seulement à l'augmentation des besoins des animaux en croissance, mais aussi à leur adaptation progressive aux rations. Dans cette figure, il faut surtout relever que la consommation avec la ration témoin est au terme de l'essai plus élevée que celles avec les rations R5 et R10. En effet, les consommations alimentaires à la sixième semaine ont été de 104,91;94,63 ; 97,70 ; 
126,33 et $137,05 \mathrm{~g}$ MS respectivement pour les rations R0, R5, R10, R15 et R20.

\section{Evolution du poids vif}

L'évolution du poids vif moyen des lapereaux est illustrée par la Figure 2. Il apparaît de l'analyse de cette figure que dans tous les lots, le poids vif croit de façon linéaire avec le temps. Cependant, bien que partis des poids très voisins, l'écart se creuse de plus en plus avec l'augmentation du taux d'incorporation de la farine de Leucaena lorsqu'on va du début à la sixième semaine de l'essai. Toutefois, il n'a été relevé aucune différence significative de poids tout au long de l'essai dans les différents traitements, et le lot témoin présente encore les meilleures performances par rapport au lot recevant la ration avec $5 \%$ de farine des feuilles de Leucaena. A la fin de l'essai, les animaux recevant $20 \%$ de L. leucocephala ont été les plus lourds (2237,50 g), suivis respectivement de ceux nourris avec $10(2134,37 \mathrm{~g}), 15$ $(2125,00 \mathrm{~g}), 0 \quad(1987,50 \mathrm{~g})$ et $5 \%$ de substitution (1968,75 g).

\section{Gain moyen quotidien}

Tout comme l'a reflété l'évolution pondérale moyenne hebdomadaire, l'analyse de la variance n'a révélé aucune différence significative $(p>0,05)$ des gains moyens journaliers en fonction des rations administrées. L'allure générale de l'évolution des gains moyens quotidiens est illustrée par la Figure 3.

En dépit des fluctuations observées, on constate de manière générale que le gain moyen quotidien augmente avec l'âge des animaux jusqu'à la cinquième semaine de l'essai ou la tendance générale est à la baisse. Toutefois, la courbe la moins variable a été celle des animaux recevant la ration avec $20 \%$ de feuilles de L. leucocephala. Le gain moyen de poids le plus élevé pour toute la durée de l'essai $(28,28 \mathrm{~g} / \mathrm{j})$ a également été obtenu avec ce même lot, et le plus faible $(21,93 \mathrm{~g} / \mathrm{j})$ avec les animaux recevant la ration $\mathrm{R} 5$, contre $23,08 \mathrm{~g} / \mathrm{j}$ pour la ration témoin.

\section{Indice de consommation}

La quantité de poids corporel produit par $\mathrm{kg}$ d'aliment consommé n'a pas été significativement influencé $(p>0,05)$ par le niveau d'incorporation de la farine des feuilles de Leucaena leucocephala. L'évolution de ce paramètre est illustrée par la Figure 4.

Il ressort de l'analyse de la Figure 4 que la tendance générale des indices de consommation est à la baisse pour les animaux nourris aux rations R0, R5, R15, alors que les lapins recevant la ration R20 présente un indice de consommation progressivement croissant, sauf entre la quatrième et la cinquième semaine. En général, les indices de consommation cumulés les plus élevés pour les six semaines de l'essai $(4,49 ; 4,32)$ ont été obtenues avec les rations $\mathrm{R} 15$ et $\mathrm{R} 20$ respectivement.

\section{Analyse économique}

Les conclusions d'une pareille étude ne saurait être valables si elles ne tiennent pas compte des sacrifices consentis, c'est-à dire de la rentabilité économique qui reste le principal objectif de toute spéculation en production animale. Le coût de production d'un kilogramme de chaque ration et de production du kilogramme vif de lapin a été estimé et présenté au Tableau 2.

Comme le montre le Tableau 2, le prix de production du kilogramme de chaque ration diminue avec l'augmentation du niveau d'incorporation de la farine des feuilles de $L$. leucocephala, probablement en raison de son prix suffisamment bas par rapport à celui du tourteau de coton. Par ailleurs, il n'existe que de légères différences entre les coûts de production du kilogramme de poids vif de lapin. Toutefois, les rations R20 et R10 restent les plus rentables, avec respectivement 633,74 et 685,14 F CFA le kilogramme de lapin à la fin de l'essai. Les meilleures performances de la ration R20 confirment une fois de plus que jusqu'à 20\% d'incorporation, les feuilles de Leucaena leucocephala n'affectent pas négativement l'indice économique de la production. 
Tableau 1 : Composition des rations expérimentales.

\begin{tabular}{lccccc}
\hline Rations & R0 & R5 & R10 & R15 & R20 \\
\hline Maïs & \multicolumn{3}{c}{ Ingrédients (kg) } & \\
Drêche & 43 & 43 & 43 & 43 & 43 \\
${ }^{*}$ CMAV (IO\%) & 26 & 26 & 26 & 26 & 26 \\
Tourteau de coton & 10 & 10 & 10 & 10 & 10 \\
Farine de feuilles de Leucaena & 20 & 15 & 10 & 5 & 0 \\
Phosphate bicalcique & 0 & 5 & 10 & 15 & 20 \\
Sulfate de fer & 0,4 & 0,4 & 0,4 & 0,4 & 0,4 \\
\hline Total & 0,6 & 0,6 & 0,6 & 0,6 & 0,6 \\
\hline & $\mathbf{1 0 0}$ & $\mathbf{1 0 0}$ & $\mathbf{1 0 0}$ & $\mathbf{1 0 0}$ & $\mathbf{1 0 0}$ \\
\hline Matière sèche & Composition chimique (en \% de matière sèche) & & \\
Protéines brutes & 92,31 & 92,38 & 92,30 & 92,45 & 92,41 \\
Cellulose brute & 18,10 & 17,60 & 17,20 & 16,60 & 16,35 \\
Matière grasse & 10,97 & 11,17 & 11,27 & 11,31 & 11,43 \\
Cendre totale & 5,96 & 4,64 & 4,20 & 3,77 & 3,64 \\
Energie métabolisable ${ }^{(1)}$ & 7,44 & 5,91 & 6,25 & 5,58 & 6,85 \\
Kcal/kg d'aliment) & 2682 & 2640 & 2600 & 2560 & 2520 \\
\hline * Concentrât minéraux azote et vitaminiques & $(10 \%)$ & & & & \\
\hline
\end{tabular}

Tableau 2 : Coût de production d'un kilogramme de chaque ration et de production du kilogramme vif de lapin.

\begin{tabular}{lcccccc}
\hline Caractéristiques & \multicolumn{5}{c}{ Rations } \\
\cline { 2 - 6 } & R0 & R5 & R10 & R15 & R20 \\
\hline Indice de consommation & 3,85 & 4,17 & 4,11 & 4,49 & 4,32 \\
\hline $\begin{array}{l}\text { Coût (F CFA) de production du } \\
\text { d'aliment }\end{array}$ & 186,70 & 176,45 & 166,70 & 156,70 & 146,70 \\
\hline $\begin{array}{l}\text { Coût (F CFA) de production du kg de } \\
\text { poids vif du lapin en fin d'essai }\end{array}$ & 718,80 & 735,80 & 685,14 & 703,58 & 633,74 \\
\hline
\end{tabular}




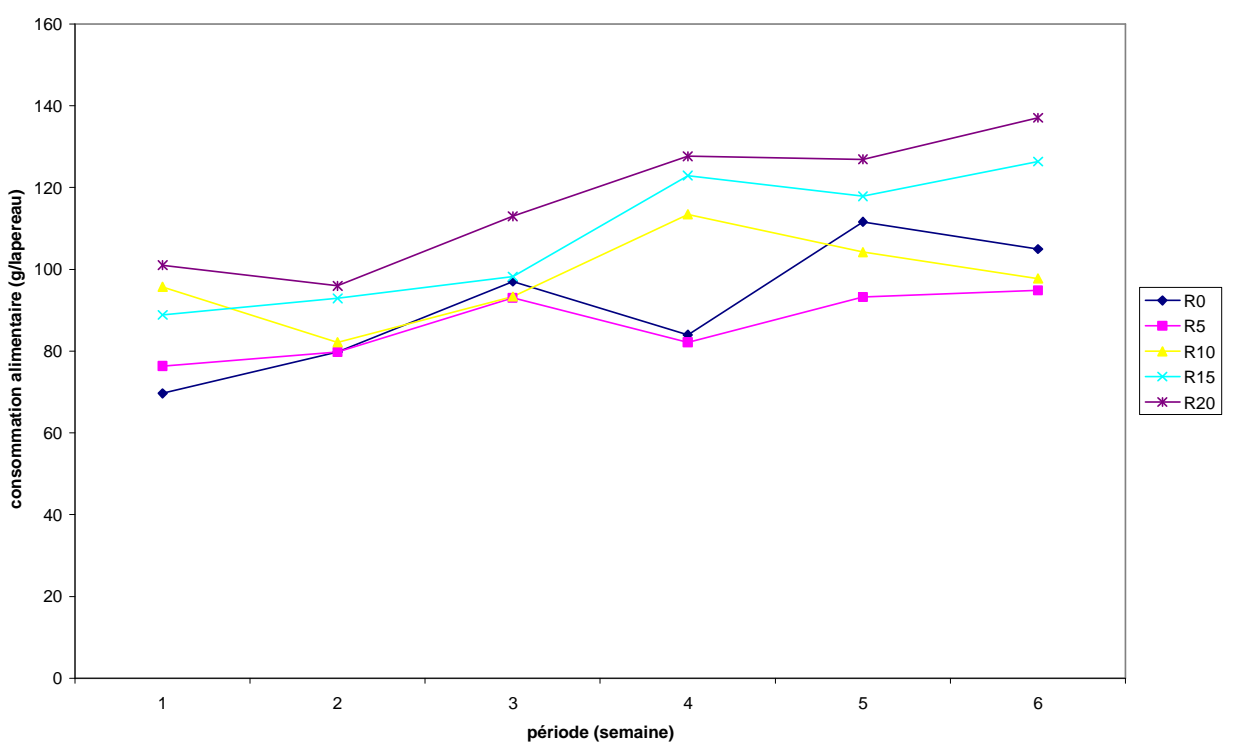

Figure 1 : Evolution hebdomadaire de la consommation alimentaire des lapereaux en fonction de la ration.

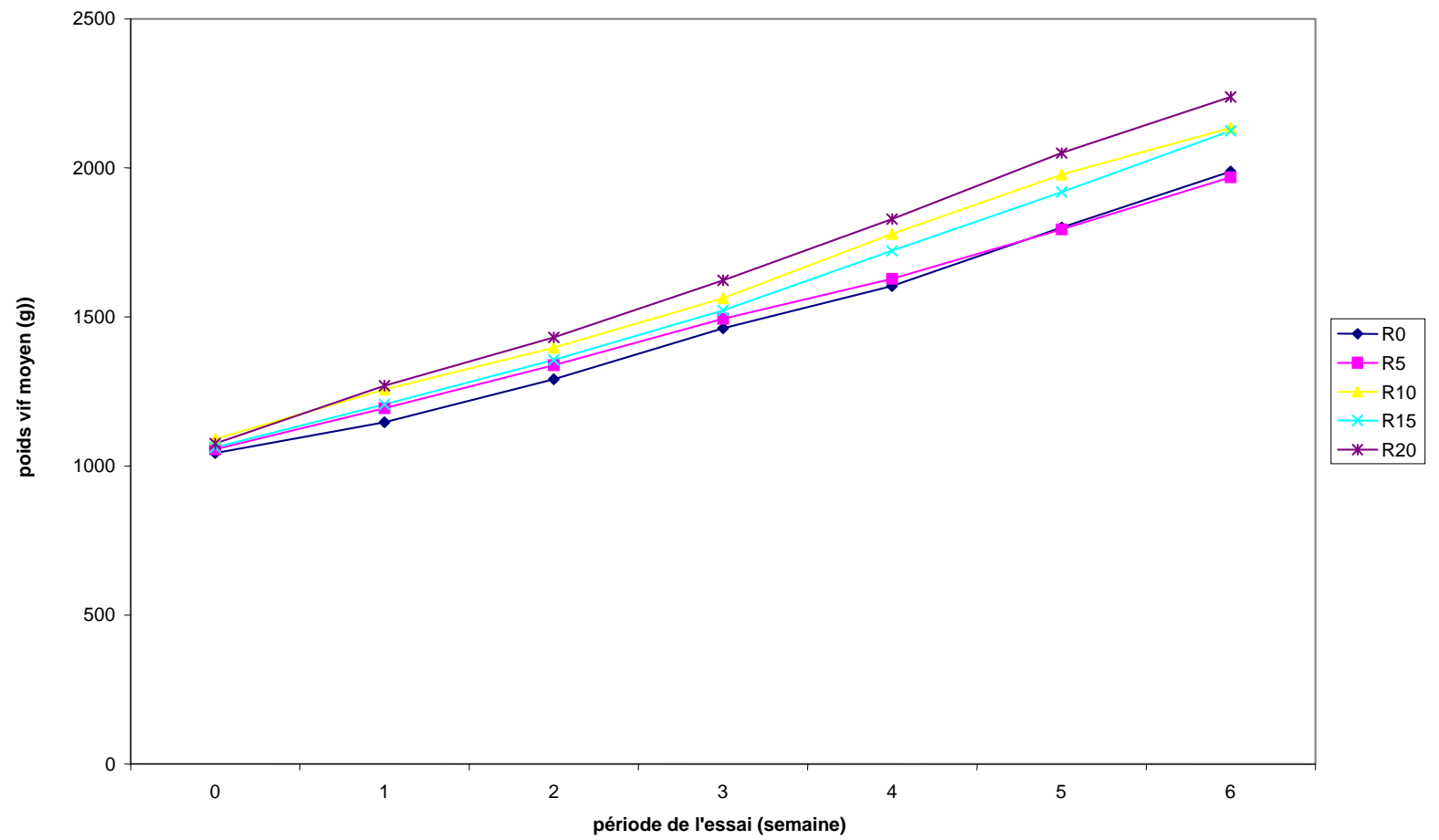

Figure 2 : Evolution hebdomadaire du poids vif des lapereaux en fonction de la ration. 


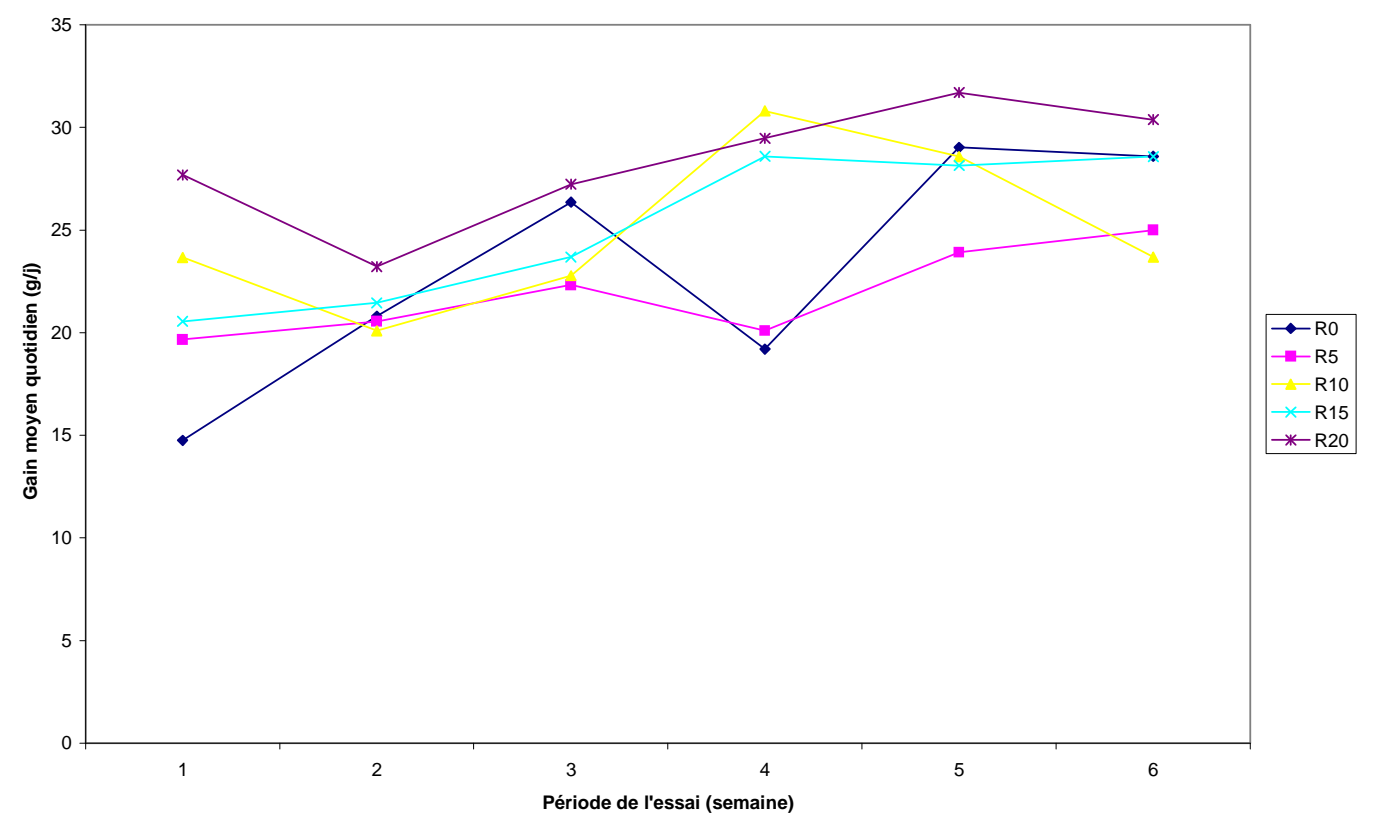

Figure 3 : Evolution hebdomadaire du Gain Moyen Quotidien des lapereaux en fonction de la ration.

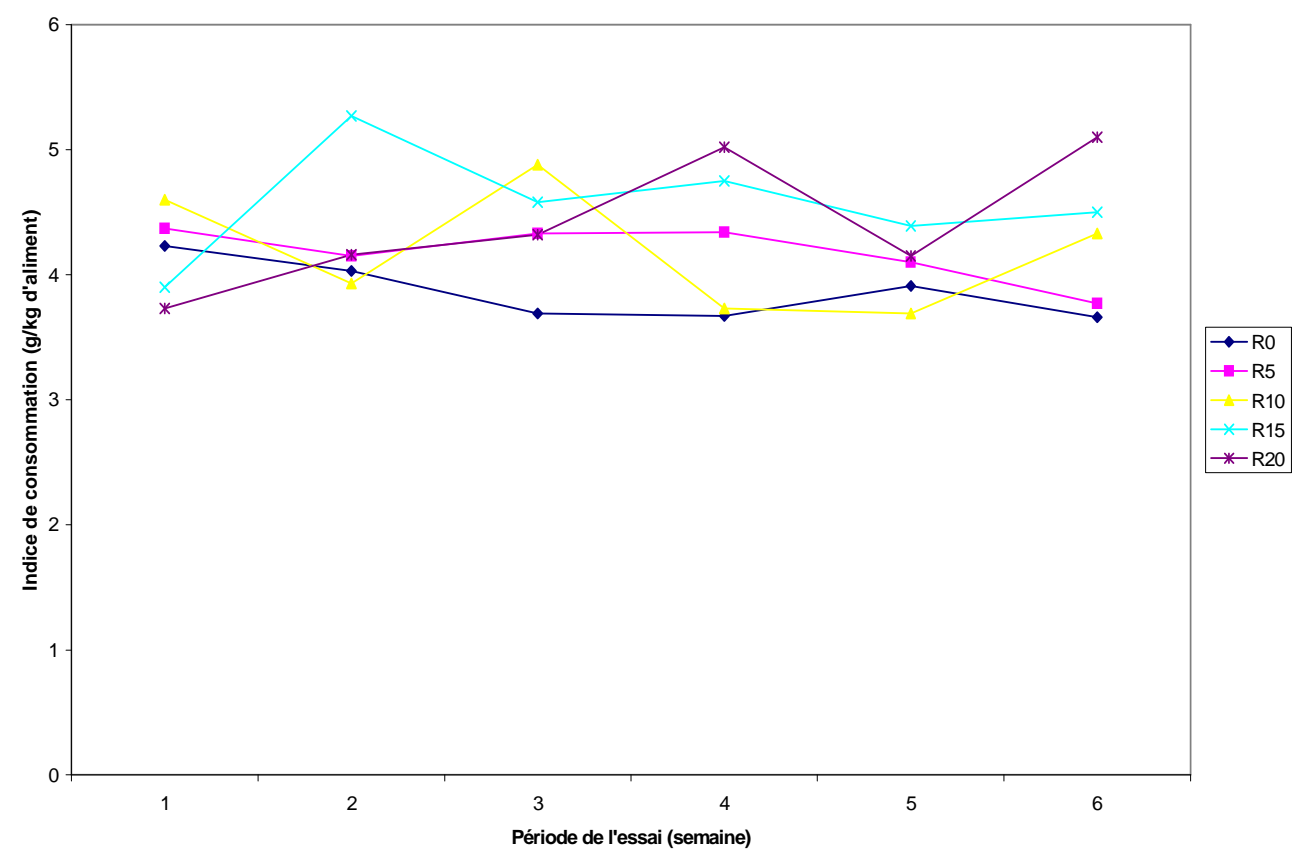

Figure 4 : Evolution de l'indice de consommation des lapereaux en fonction de la ration. 


\section{DISCUSSION}

L'évolution de la consommation alimentaire avec l'augmentation du taux d'incorporation des feuilles de $L$. leucocephala est en conformité avec les travaux de Lamidi et Akilapa (2013). Toutefois, ces performances restent inférieures aux standards $(120-160 \mathrm{~g} / \mathrm{j})$ signalés par Lebas (1983). Des explications pourraient dériver de la race, du climat, de la conduite de l'élevage et même de la forme de présentation de la ration qui, lorsqu'elle n'est pas granulée, affecte négativement la prise alimentaire. Cependant, ce niveau de consommation alimentaire n'est pas en conformité avec celui rapporté par Mtenga et Laswai (1994) qui a conduit à un faible taux de croissance et d'efficacité alimentaire.

Les résultats de l'évolution du poids vif avec le taux d'incorporation sont toutefois inférieurs à ceux obtenus par Ramchurn et al. (2000) en Mauritanie. Les résultats de gain de poids obtenus dans cet essai se rapprochent de ceux de Djellal et al. (2006) en milieu paysan Algérien. Ces gains de poids sont cependant inférieurs aux normes de $30-33 \mathrm{~g} / \mathrm{j}$ rencontrées dans la littérature Defang et al. (2011). Ces faiblesses pourraient s'expliquer d'une part par la faible consommation alimentaire générale, d'autre part par le faible potentiel génétique des animaux.

\section{Conclusion}

Au terme de cette étude, nous pouvons dire en guise de conclusion que: la consommation alimentaire augmente avec le niveau d'incorporation des feuilles de Leucaena dans les rations des lapereaux; l'augmentation du taux d'incorporation n'a pas significativement influencé l'évolution du poids vif ni le gain moyen quotidien, bien que les lapereaux recevant des taux de 15 et $20 \%$ de farine de feuille de Leucaena leucocephala semble plus favorisés; l'indice de consommation a été meilleur chez les animaux recevant de faible taux de substitution du tourteau de coton par les feuilles de Leucaena; le coût de production d'un kilogramme d'aliment baisse progressivement avec l'augmentation $\mathrm{du}$ niveau d'incorporation de la farine de Leucaena.

De manière générale, les lapereaux ont semblé mieux se comporter avec la ration R20 dans les conditions de cette étude. Ceci laisse croire qu'on pourrait encourager l'utilisation de la farine de feuilles de L. leucocephala à des taux au moins égaux à $20 \%$. Toutefois, d'autres investigations sont nécessaires pour déterminer le niveau optimal d'incorporation de Leucaena en évitant les effets néfastes de la mimosine que contiendraient ces feuilles.

\section{RÉFÉRENCES}

Aregheore EM, Perera D. 2004. Effect of supplementation of basal diet of maize stover with Erythrena variegata, Gliciridia sepium or Leucaena leucocephala on feed intake and digestibility by goats. Tropical Animal Health and Production, 36: 175-189.

Bergaoui R. 1992. L'élevage du lapin en Tunisie peut contribuer à résoudre le problème de déficit en viande du pays. Options Méditerranéennes - Série Séminaires - $\mathrm{n}^{\circ} 17,23-32$.

Defang HF, Defang A, Teguia F, Ngoula AT, Niba A, Kenfack JR, Kana Tendongkeng F, Tchoumboue J, Ndessap J. 2011. Effect of incorporating maize cobs (Zea mais) as a source of crude fiber on the growth performance of grower rabbits. Sciences Agronomiques et Développement, 6(1): 5 - 11.

Djellal F, Mouhous A, Kadi SA 2006. Performances de l'élevage fermier du lapin dans la région de Tizi-Ouzou, Algérie. Livestock Research for Rural Development. 18: Article \#100. Retrieved December 4, 2012, from http://www. lrrd.org/lrrd18/7/djel18100.htm

Harris DJ, Cheeke PR, Patton NM. 1983. Feed preferences studies with rabbits fed 
fourteen different fresh greens. Journal of Applied Rabbit Research, 6: 120-124.

Lamidi WA, Akilapa TP. 2013. Performance characteristics of rabbits fed with Leucaena leaf meal diet in natural housing system. Agricultural science Research Journal, 144-151, http://www.resjournals.com/ARJ

Lebas F. 1983. Small-scale rabbit production: Feeding and management systems. Wild Animal Review, 46:11-17.

Mtenga LA, Laswai GH. 1994. Leucaena leucocephala as feed for rabbits and pigs: detailed chemical composition and effect of level of inclusion on performance.
Ecology Management Journal, 54: 249257.

Ramchurn R, Dullull ZB, Ruggoo A, Raggoo J. 2000. Effects of feeding star grass (Cynodon plectostachyus) on growth and digestibility of nutrients in the domestic rabbit. Livestock Research for Rural Development, 12(2): http://www.cipav. org.co/lrrd/lrrd12/2/ram122.htm

Jabbar MA, Reynolds L, Larbi A, Smith J. 1997. Nutritional and economic benefits of Leucaena and Gliciridia as feed supplements for small ruminants in humid West Africa. Tropical Animal Health and Production, 29: 35-4. 